\title{
Oral administration of tannins and flavonoids in children with acute diarrhea: a pilot, randomized, control-case study
}

\author{
Marina Russo, Vincenzo Coppola, Eleonora Giannetti, Roberta Buonavolontà, Antonio Piscitelli \\ and Annamaria Staiano*
}

\begin{abstract}
Background: $A G$ is the most common cause of pediatric consultations among children between 2 and 5 years of age and it still leads to high mortality and morbidity. Its management is based on rehydration therapy, but this treatment is not effective in reducing duration of diarrhea. For this reason, other safer and less expensive interventions, which could be added to oral rehydration therapy, are of great interest.

Methods: A pilot, randomized, case-controlled trial was conducted in 60 children affected by AG ( $<7$ days) with mild-moderate dehydration, according to WHO recommendations, from 1 year to 17 years old. Patients were divided into 2 Groups: Group 1 consisting of 30 children treated with Actitan F and standard oral rehydration (SOR); Group 2 consisting of 30 children who received only SOR. Both groups received treatment for seven days, respectively. Patients of Group 1 stopped for their own choice, SOR after the first $24 \mathrm{~h}$ and continued only with Actitan F.

Results: After $24 \mathrm{~h}$ of treatment, the median number of stools was 3.5 for Group 1, and 4 for Group 2. In Group 1 the difference between the number of stools at baseline $(n=5)$ and after $24 \mathrm{~h}$ of treatment $(n=3.5)$ was significant $(p<0$. 0001). At the end of treatment, the median duration of diarrhea in Group 1 was 5 days, compared with 4 days in the Group 2, this difference was not statically significant ( $p$ 0.48).

Conclusions: Oral administration of Actitan F associated with SOR seems safe and effective treatment in shortening the duration of AG in children. Further studies confirming these data are needed.
\end{abstract}

Trial registration: NCT03356327 (retrospectively registered).

Keywords: Acute gastroenteritis, Treatment, Tannins, Flavonoids

\section{Background}

Acute gastroenteritis (AG) is the most common cause of pediatric consultations among children between 2 and 5 years of age [1]. AG in children still leads to high mortality and morbidity [2].According to current European guidelines, the main treatment for AG is the oral rehydration with anhypo-osmolar solution and regular feeding without dietary changes, including milk consumption. However, these treatment, does not reduce severity and duration of symptoms. Considering the burden of AG to children and the high cost for healthcare system, effective

\footnotetext{
* Correspondence: staiano@unina.it

Department of Translational Medical Science, Section of Pediatrics, "Federico II" University of Naples, Via S. Pansini, 5, 80131 Naples, Italy
}

and less expensive interventions, that could be added to the effect of oral rehydration therapy, are of great interest [3].

According to the European Society of Gastroenterology and Hepatology (ESPGHAN) guidelines for diarrhoea [4], probiotics, with evidence of efficacy such as $S$. boulardii and L. rhamnosus GG, can be considered as a supplement to hypo-osmolar solution. It is known that probiotics reduce the mean duration of diarrhoea by about $24 \mathrm{~h}$, but their efficacy on stool amount is not described [4]. Although the grade of recommendation is very strong, quality of evidences is still low [5]. Other preparations used for treatment of diarrhoea include oral administration of immunoglobulins, antiperistaltic and antisecretory agents, such as various preparations of bismuth subsalicylate, cholestyramine and loperamide [6-10]. Use of

(c) The Author(s). 2018 Open Access This article is distributed under the terms of the Creative Commons Attribution 4.0 International License (http://creativecommons.org/licenses/by/4.0/), which permits unrestricted use, distribution, and 
antiperistaltic drugs is not effective and can cause serious side effects in children, including lethargy, seizures, Reye's syndrome, paralytic ileus and respiratory depression [11-13]. Use of loperamide, one of the most common antiperistaltic agents, is not recommended in young children and infants.

In conclusion, an ideal antidiarrheal agent should have a high index of safety even when used without close professional supervisions, and should be compatible with oral rehydration solutions, treating effectively diarrhea of any etiology with moderate cost. During last years, several randomized and controlled studies showed the efficacy of tannin-rich carob pod for the treatment of acute-onset diarrhea [10]. In 2003,Subbotina et al. demonstrated that tormentil root extract, which has high content of tannins, reduces the duration of rotavirus diarrhea compared with the control group in a pediatric population $(P<0.0001)$ [5].They also demonstrated that in the group treated with tormentil root extract, 8 of 20 children (40\%) were diarrhea-free $48 \mathrm{~h}$ after admission to the hospital, compared with 1 of 20 in control group (5\%, $P<0.0001)[5]$.

In this pilot study we aimed to evaluate the efficacy and the compliance of a treatment with Actitan-F, a natural molecular complex of tannins (from Agrimony and Tormentil) and flavonoids (Chamomile), associated with SOR, in a pediatric population of children affected by AG.

\section{Methods}

We included 60 children (mean age: 3.1 yrs., range 0.312 years) with a diagnosis of AG, referred between April and July 2017 to the Department of Translational Medicine, section of Pediatric, University of Naples Federico II.

Patients enrolled were children from 3 months to 12 years old, with a diagnosis of acute diarrhoea appeared less than 7 days before the admission, capability to oral rehydration, mild to moderate dehydration. Patients with diarrhoea over 7 days, serious somatic pathology and severe dehydration were excluded.

The study was approved by the Institutional Review Board of the University of Naples "Federico II" with the protocol number 25/17. At admission, written informed consent was obtained from participants' parents and from all patients older than 10 years. At first visit, a medical history was collected by one of the authors and all patients underwent clinical evaluation, including body weight and body temperature.

Frequency of bowel movements, stool consistency measured through the Bristol Stool Form Scale (BSFS) and other associated gastrointestinal symptoms, including nausea, vomiting, abdominal pain and rectal bleeding, were accurately recorded. The BSFS is the most commonly standardized instrument used to rate stool consistency in children [14]. On admission, the degree of dehydration was clinically determined for each patient, based on WHO recommendations and data were recorded on a scale from 1 to 3 ( 1 for mild or $<5 \%$; 2 for moderate or 5 to $10 \%$; 3 for severe or $10 \%$ and more) [15].

All enrolled children were randomly divided into two groups: Group 1 was treated with Actitan F and standard oral rehydration (SOR) and Group 2 was treated with SOR only ad libitum for 7 days (Fig. 1) SOR is a reduced osmolarity oral solution $(50 / 60 \mathrm{mmol} / \mathrm{L} \mathrm{Na})$, which is the first line therapy recommended by ESPGHAN guideline for Acute Diarrhea [16]. Actitan F, instead, was orally administrated at dose of 1 sack every $4 \mathrm{~h}$, maximum 4 sacks/ day for 7 days. Caregivers were instructed to administer the daily dose after mixing the contents of the sachet with a small amount of water. The study products used in this trial were donated by Aboca ${ }^{\circ}$ Società Agricola SpA., Località Aboca, 20, 52,037 Sansepolcro (AR) - Italy.

At home, all parents had to fulfil a daily diary to record number and consistency of stools, presence of fever, vomiting and children compliance with the therapy. During the final visit, scheduled after 7 days, the interim history was assessed, daily diaries were reviewed and discussed, and a physical evaluation was performed.

\section{Outcomes}

The primary outcome was to evaluate the efficacy of a treatment with Actitan-F, a natural molecular complex of tannins (from Agrimony and Tormentil) and flavonoids (Chamomile) added to SOR compared to SOR in a paediatric population of children affected by AG.

The secondary outcome was to assess safety, tolerability and compliance of the study products for short-term treatment.

\section{Endpoints}

The primary endpoint was the duration of diarrhoea, defined as the number of stools after $24 \mathrm{~h}$ of treatment or the time needed to normalize number and consistency of stools (compared with the period before the onset of diarrhoea). Secondary endpoint were the evaluation of vomiting, body weight, possible need of hospitalization, compliance to therapy.

\section{Statistical analysis}

Data were analysed using SPSS version 17.0 (SPSS Inc., Chicago, Illinois, USA) for descriptive and frequency results and presented as median and range for quantitative variables and percentiles for categorical variables. Significance was defined as $P$ value $<0.05$. Comparisons between the two groups used the Fisher's exact test for categorical variables and the Mann-Whitney $U$ test for quantitative variables. $\mathrm{X}^{2}$ test was used to 


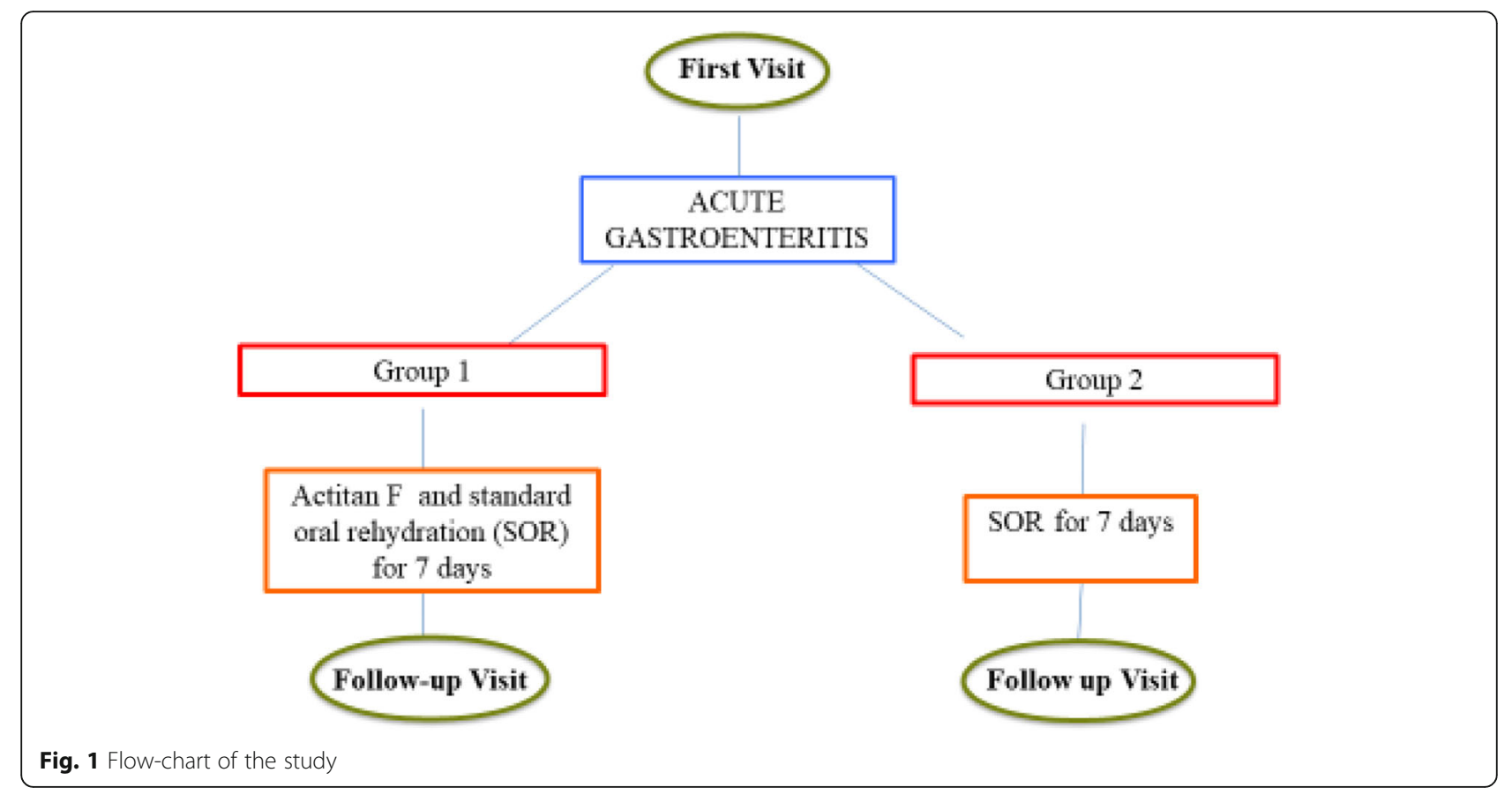

determine patients' acceptance of the products (Acti$\tan \mathrm{F}$ and SOR) we tasted.

\section{Results}

All patients completed the study. Socio-demographic and clinical characteristics of the study population are showed in Table 1. At baseline, a mean of defecation/ daily was 5 in both groups (ranged from 3 to 8 in Group 1 and from 2 to 10 in Group 2) ( $p=0.63)$. The median number of stools after $24 \mathrm{~h}$ was 3.5 in Group 1 and 4 in Group 2. The difference in the number of stools the first day of treatment between the two groups was not statistically significant $(p=0.4)$; however, the difference between the number of stools at baseline and after $24 \mathrm{~h}$ of treatment was significant $(\mathrm{p}=<0.0001)$ in the group treated with Actitan F. After $24 \mathrm{~h}$ of treatment, we found that the most of children $(23 / 30,76 \%)$ in Group 1, by their own choice, interrupted the assumption of SOR and continued to take only Actitan F. At the end of treatment, in Group 1 the primary endpoint was reached after 5 days while in Group 2 after 4 days, this difference was not statistically significant ( $p=0.48$ ) (Fig. 2$)$. We also showed improved stool consistency from baseline to $24 \mathrm{~h}$ in the two groups, from liquid stools in 89.7 and $86.7 \%$ for Group 1 and Group 2, respectively, to 60 and $73.3 \%(p=0.34)$. In particular, regarding stool consistency, we found that at day 6 in Group $176.9 \%$ of patients reported formed stools compared with $50 \%$ in Group $2(p=0.028)$. The presence of vomiting at baseline was $73.3 \%$ (22 children of 30 ) for Group 1 and 43.3\% (13 children of 30) for the Group 2; at $24 \mathrm{~h}$ vomiting was present in $46.6 \%$ of the Group 1 and $16 \%$ of the Group 2 with no statistical significant difference. Bloody diarrhoea was found in only 10 and $6 \%$ of patients at baseline for Group 1 and Group 2, respectively; at the end of treatment, no patient presented blood in the stool in both groups. As for weight and fever, we did not

Table 1 Demographics and Clinical characteristics at baseline

\begin{tabular}{|c|c|c|c|c|}
\hline & Overall $(N=60)$ & Group $1(N=30)$ & Group $2(N=30)$ & $p$-value \\
\hline Median age (range), years & $3.1(0.3-12.7)$ & $4.1(0.5-12.1)$ & $2.7(0.3-12.7)$ & 0.23 \\
\hline Starting date of diarrhea before consultation in days (range) & $1(1-4)$ & $1(1-4)$ & $1(1-3)$ & 0.32 \\
\hline Median number of stools/day at baseline (range) & $5(2-10)$ & $5(3-8)$ & $5(2-10)$ & 0.63 \\
\hline Consistency of stools at baseline (\%) & & & & 0.35 \\
\hline Watery & $81.4 \%$ & $86.2 \%$ & $76.7 \%$ & \\
\hline Soft & $18.6 \%$ & $13.8 \%$ & $23.3 \%$ & \\
\hline Vomiting at baseline (median and range) & $1.5(0-23)$ & $3(0-23)$ & $0.5(0-7)$ & 0.017 \\
\hline Fever $(\%)$ & $50.0 \%$ & $50.0 \%$ & $50.0 \%$ & 1.0 \\
\hline
\end{tabular}


Number of stools/day

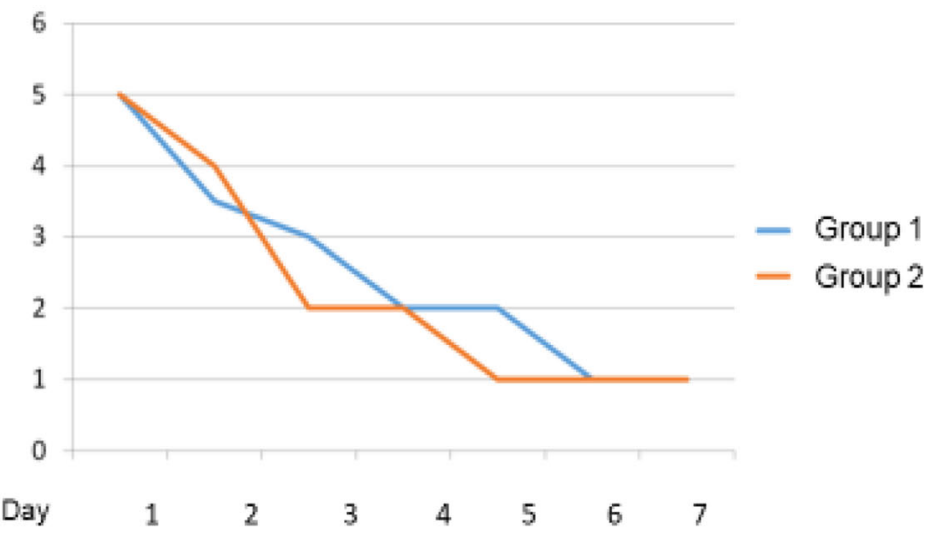

Fig. 2 Primary Endpoint: Bowel movements/ day

find any statistically significant difference between the groups. No one was hospitalized.

\section{Compliance and patient acceptance of treatment} Concerning compliance with therapy, 8/30 children (26\%) in Group 1 and 10/30 (30\%) in Group 2 refused to take Actitan F and SOR, respectively $(p=0.77)$ (Fig. 3). Regarding the perception of the treatment efficacy, parents of children in Group 1 evaluated the Actitan F treatment very useful in $50 \%$ of cases, while parents of patients in Group 2 evaluated SOR quite effective in $68 \%$ of cases, this difference was statistically significant $(p<0.0001)$. Similar trend was present in the perception of improvement after $24 \mathrm{~h}$, where we found a tendency to significance in Group 1 with respect to Group $2(p=0.07)$. No adverse events in both groups were reported during the study.

\section{Discussion}

In this pilot study, we investigated the therapeutic efficacy of a medical device made of natural complex

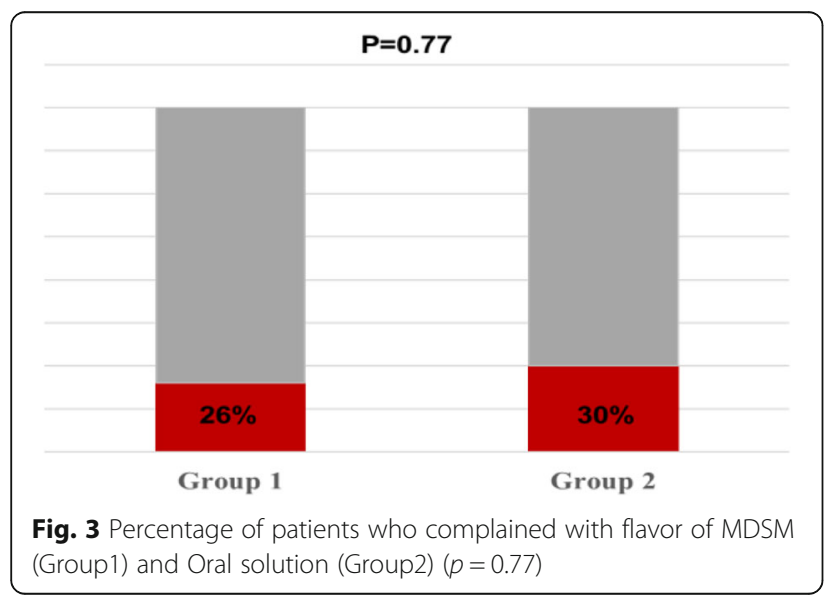

substances in the treatment of children with AG. The positive clinical effect exerted by this new product on diarrhoea could be related to the tannins. Their action, indeed, is essentially focused on the intestinal mucosa, favouring its rapid recovery. More specifically, when tannins meet mucous membranes, they aspecifically interact with those membranes by making them more tight and less permeable; this process is called "astringency". This feature increases protection to the subadjacent layers of mucosa from the microorganisms and irritant chemicals [17].

The efficacy and safety of a similar product in treatment of acute diarrhoea in pediatrics have been already demonstrated during the last years. In 2009 Carretero et al. observed a significant decrease in the number of stools and an improvement in stool consistency in patients treated with oral rehydration solution (SOR) and gelatin tannate vs. patients treated only with SOR [18]. Loeb et al. showed that patients receiving a product containing tannate had a normalization of bowel movements, body temperature, weight, and ceased to vomit much faster than those receiving placebo [10].

Plein and colleagues studied the effect of tannate on diarrhoea in patients with Crohn's disease [19]. The results obtained demonstrated that there was a significant reduction in bowel movement frequency at the end of treatment. Another clinical experience was reported by Ziegenhagen and colleagues, who showed the better efficacy and safety profile of tannin salts versus activated charcoal. Moreover, in the group receiving tannin salts, the frequency of abdominal pain was lower than in the activated charcoal group (50 versus 82\%) [20].

Although our study protocol included the administration of Actitan F plus SOR for a week in Group 1 and SOR alone in Group 2, most of the patients in the first Group interrupted the assumption of oral rehydration 
after the first day of treatment. We can explain this choice considering that after $24 \mathrm{~h}$ of combined treatment the reduction in number of stools in Group 1 was significantly different than at baseline ( $p<0.0001)$ ( 5 vs. 3.5 stools/day).

Due to the high perception of symptom improvement reported by parents of children in Group 1 after the first day of treatment, we can speculate that the rapid improvement of clinical condition may have induced parents to stop earlier the oral solution and continue only with Actitan F.

As a matter of fact, our results showing that the mean duration of diarrhea in both groups after 7 days of therapy was not statistically significant, should be interpreted in the light of what we have just described.

Regarding stool consistency, we showed no statistically significant differences between the two groups, however at day 6 of treatment we found that $76.9 \%$ of patients in Group 1 reported formed stools compared with $50 \%$ in Group 2, this data showed a tendency to significance with a $p=0,028$, maybe do to the small number of patients enrolled. We can explain this better result on stool consistency comparing to SOR alone, knowing that it has already been demonstrated that tannins and flavonoids exerts their action by restoring the physiological function of the intestine barrier and have astringent properties [17].

Moreover, in our study any adverse event in children were observed in both groups, and this confirms the safety of tannins in the pediatric population. Compliance with administration of Actitan F was good; every patient received $75 \%$ of doses according to the patient's weight. This result confirms a good ease of administration.

In contrast to other studies, we did not perform stool cultures at baseline [21, 22]. Nevertheless, ESPGHAN does not recommend performing stool cultures for acute gastroenteritis in primary healthcare, and we did not aim to evaluate the impact of tannins based on the different aetiologies of diarrhoea.

\section{Conclusion}

Therefore, we can conclude that Actitan F in association with SOR, may offer a safe and cost-effective approach to AG in infants and children. However, further studies and large trials are required. Because this study showed promising results, it would be worthwhile to perform a large randomized control trial to unravel the efficacy of the Actitan F plus SOR in children with AG.

\section{Abbreviations}

AG: Acute gastroenteritis; SOR: standard oral rehydration

Funding

Aboca ${ }^{\circledR}$ Società Agricola SpA., Località Aboca, 20, 52037 Sansepolcro (AR) - Italy.

Availability of data and materials

Data will not be shared in accordance to the Department's policy.

\section{Authors' contributions}

MR performed data acquisition, analysed the data, wrote the first draft of the manuscript, and approved the final version of the paper. VC performed data acquisition, critically revised the manuscript and approved the final version of the paper. VC, RB and AP performed data acquisition, critically revised the manuscript and approved the final version of the paper. EG designed the research study, analyzed the data, and approved the final version of the paper. AS designed the research study, critically revised the manuscript and approved the final version and the submission.

\section{Ethics approval and consent to participate}

The study was approved by the Independent Ethics Committee of the "Federico II" University of Naples (reference number: 25/17).

\section{Competing interests}

The authors declare that they have no competing interests.

\section{Publisher's Note}

Springer Nature remains neutral with regard to jurisdictional claims in published maps and institutional affiliations.

Received: 30 November 2017 Accepted: 9 May 2018

Published online: 04 June 2018

\section{References}

1. Van Damme P. Multicenter prospective study of the burden of rotavirus acute gastroenteritis in Europe, 2004-2005: the REVEAL study. J Infect Dis. 2007;195(Suppl 1):S4-16.

2. Ogilvie I. Burden of community-acquired and nosocomial rotavirus gastroenteritis in the pediatric population of Western Europe: a scoping review. BMC Infect Dis. 2012;12:62.

3. Szajewska H. Probiotics in gastrointestinal diseases in children: hard and not-so-hard evidence of efficacy. J Pediatr Gastroenterol Nutr. 2006:42:454-75.

4. Guarino A. Probiotics as prevention and treatment for diarrhea. Curr Opin Gastroenterol. 2009;25(1):18-23.

5. Subbotina Maria D. Effect of oral administration of tormentil root extract (Potentilla tormentilla) on rotavirus diarrhea in children: a randomized, double blind, controlled trial. Pediatr Infect Dis J. 2003;22:706-10.

6. Madkour AA. Cholestyramine in acute infantile diarrhea: a double-blind, placebo controlled clinical trial in hospitalized infants. Alexandria. J Pediatr. 1990;4:319-32.

7. Madkour AA. Smectite in acute diarrhea of children: a double-blind placebocontrolled clinical trial. Gastroenterology. 1991;100:A227.

8. Kaplan MA, Prior MJ, McKonly Kl, et al. A multicenter randomized controlled trial of liquid loperamide product versus placebo in the treatment of acute diarrhea in children. Clin Pediatr. 1999:38:579-91.

9. Ebina T. Prevention of rotavirus infection with cow colostrum containing antibody against human rotavirus. Lancet. 1983;2:1029-30.

10. Loeb H. Tannin-rich carob pod for the treatment of acute-onset diarrhea. J Pediatr Gastroenterol Nutr. 1989:8:480-5.

11. Elaraby II. The use of chlorpromazine as an antisecretory agent in acute diarrhea of childhood. Alexandria J Pediatr. 1989;3:1-8.

12. Kassem AS. Loperamide in acute childhood diarrhoea: a double blind controlled trial. J Diarr Dis Res. 1983;1:10-6.

13. Kassem AS. Effect of antibiotics on the duration of diarrhea and speed of rehydration. Gazette Egyptian Paediatr Assoc 1983;31:117-118.

14. Lane MM. Reliability and validity of a modified Bristol stool form scale for children, J Pediatr. 2011;159:437-41.e1.

15. World Health Organization. The treatment of diarrhea: A manual for physicians and other senior health workers; 2005.

16. Guarino A. European Society for Pediatric Gastroenterology, hepatology, and nutrition/European Society for Pediatric Infectious Diseases EvidenceBased Guidelines for the Management of Acute Gastroenteritis in children in Europe: update 2014. JPGN. 2014;59(Issue 1):132-52.

17. Mills S, Bone K. The essential guide to herbal safety. UK: Churchil Livingstone; 2005.

18. Esteban Carretero J. A comparative analysis of ORS (oral rehydration solution) vs. ORS + gelatin tannin in two cohorts of pediatric patients with acute diarrhea. REV ESP ENFERM DIG (Madrid). 2009;101(1):41-8. 
19. Plein K. Treatment of chronic diarrhea in Crohn disease. A pilot study of the clinical effect of tannin albuminate and ethacridine lactate. Fortschr Med. 1993;111:114-8.

20. Ziegenhagen DJ. Traveler's diarrhea in Turkey. Prospective randomized therapeutic comparison of charcoal versus tannin albuminate/ethacridine lactate. Med Klin. 1992;87:637-9.

21. Salazar-Lindo E. Racecadotril in the treatment of acute watery diarrhea in children. N Engl Med J. 2000;343:463-7.

22. Cezard J. Efficacy and tolerability of Racecadotril in acute diarrhea in children. Gastroenterology. 2001;120(799-805):13.

Ready to submit your research? Choose BMC and benefit from:

- fast, convenient online submission

- thorough peer review by experienced researchers in your field

- rapid publication on acceptance

- support for research data, including large and complex data types

- gold Open Access which fosters wider collaboration and increased citations

- maximum visibility for your research: over $100 \mathrm{M}$ website views per year 